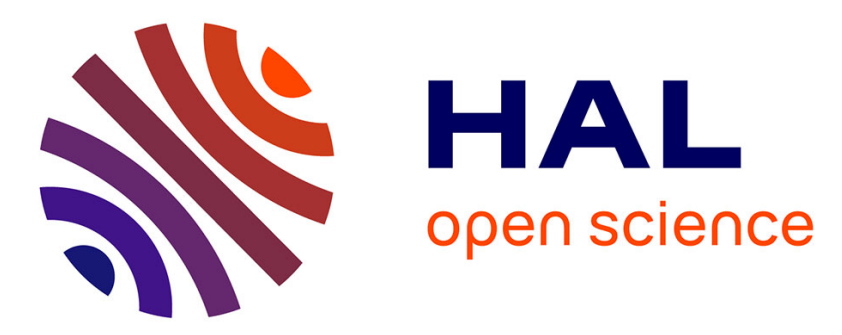

\title{
Performances de la méthode des moments dans l'étude de petits amas d'atomes métalliques
}

\author{
M. Leleyter, P. Joyes
}

\section{To cite this version:}

M. Leleyter, P. Joyes. Performances de la méthode des moments dans l'étude de petits amas d'atomes métalliques. Journal de Physique Lettres, 1976, 37 (11), pp.303-308. 10.1051/jphyslet:019760037011030300 . jpa-00231298

\section{HAL Id: jpa-00231298 https://hal.science/jpa-00231298}

Submitted on 1 Jan 1976

HAL is a multi-disciplinary open access archive for the deposit and dissemination of scientific research documents, whether they are published or not. The documents may come from teaching and research institutions in France or abroad, or from public or private research centers.
L'archive ouverte pluridisciplinaire HAL, est destinée au dépôt et à la diffusion de documents scientifiques de niveau recherche, publiés ou non, émanant des établissements d'enseignement et de recherche français ou étrangers, des laboratoires publics ou privés. 


\title{
PERFORMANCES DE LA MÉTHODE DES MOMENTS DANS L'ÉTUde DE PETITS AMAS D'ATOMES MÉTALLIQUES
}

\author{
M. LELEYTER et P. JOYES \\ Laboratoire de Physique des Solides associé au C.N.R.S., \\ Bât. 510, Université Paris-Sud, 91405 Orsay Cedex, France
}

(Reçu le 16 juillet 1976, accepté le 5 août 1976)

\begin{abstract}
Résumé. - Nous étendons la méthode des développements en moments de la densité d'états, utilisée jusqu'à présent pour le solide, au cas d'amas finis ( $\sim 50$ atomes) d'atomes à un électron $\mathrm{s}$ de valence (groupe I). Les performances de cette technique se montrent remarquables pour l'étude des stabilités relatives de diverses structures de hautes symétries de ces amas (icosédrique ou cubique) ainsi que pour la détermination de la limite inférieure du spectre de niveaux. La méthode, plus intéressante que celle de Hückel car plus rapide, peut être étendue avec bénéfice aux amas de quelques centaines d'atomes.
\end{abstract}

\begin{abstract}
We give an extension of the moment development method, used untill now for solids, to the case of finite clusters ( $\sim 50$ atoms) of group I atoms. The technique is very successful in studying relative stabilities of different high symmetry structures (icosahedral or cubic) of the clusters, and also for the determination of the lower limit of the level spectrum. This method is more interesting than the Hückel one because it is quicker and can usefully be extended to clusters of several hundred atoms.
\end{abstract}

1. Introduction. - La structure électronique d'agrégats de quelques atomes métalliques commence à être étudiée de manière approfondie car ce type d'amas présente un intérêt certain en particulier du point de vue des propriétés physiques telles que susceptibilités magnétiques ou capacités calorifiques. On peut penser en effet qu'ils se situent dans une progression logique de l'atome au solide et certains auteurs ont d'ailleurs déjà tenté d'extrapoler des propriétés des solides à partir de celles d'amas de quelques atomes [1,2].

Toutefois ces propriétés doivent être déterminées à partir de la densité des états d'énergie des agrégats. Or ces derniers, de dimensions finies, sont caractérisés fondamentalement par un spectre discret de niveaux d'énergie (effet de taille quantique).

Jusqu'à ces derniers temps, la plupart des auteurs $[3,4]$ sont partis d'un spectre de niveaux donné a priori, avec par exemple une loi de répartition au hasard des niveaux dans l'échelle des énergies, ce qui leur a permis par des calculs de Mécanique Statistique, d'atteindre alors les propriétés recherchées.

Une autre manière de procéder est de calculer complètement le spectre de niveaux en utilisant des méthodes empruntées à la Chimie Quantique, en particulier la méthode des orbitales moléculaires qui s'est révélée extrêmement fructueuse pour les agrégats de quelques atomes (10 atomes ou moins) [5]. Toutefois, lorsque les amas comportent quelques dizaines, voire quelques centaines d'atomes, cette technique de calcul, même sous la forme approchée que constitue la méthode de Hückel [6], tend à devenir assez lourde puisqu'il s'agit principalement de diagonaliser une matrice dont les dimensions sont au minimum égales au nombre d'atomes $N$ de l'amas.

Il est donc intéressant de rechercher une approximation à cette méthode qui permettrait de simplifier le calcul de manière à pouvoir envisager l'étude de plus gros amas, de quelques centaines d'atomes par exemple. La méthode du développement en moments de la densité d'états, utilisée jusqu'à présent pour le solide [7,8], parait remplir cette condition et permet en particulier d'approcher les résultats de la méthode de Hückel avec une précision contrôlable.

Nous nous proposons ici d'évaluer les performances de cette méthode lorsqu'on l'applique aux agrégats de quelques dizaines d'atomes d'éléments à un électron $\mathrm{s}$ de valence par atome. Nous essaierons en particulier de comparer les énergies de cohésion de différentes structures de haute symétrie et de déterminer la précision obtenue sur les niveaux d'énergie [5].

Nous allons commencer par rappeler rapidement comment on peut obtenir une densité d'états à partir des moments des agrégats.

2. Présentation de la méthode. - Cette méthode est très utilisée en Physique des Solides et on peut en trouver un exposé dans de nombreux travaux $[7,8,9]$. 
Il est très rare que l'on sache obtenir une expression analytique de la densité d'états à tous les ordres ; le procédé utilisé est donc de calculer les moments jusqu'à l'ordre le plus élevé possible, puis d'en déduire une densité d'états approchée $n_{\mathrm{ap}}(E)$. On peut dans cette dernière phase faire en sorte que $n_{\mathrm{ap}}(E)$ soit une fonction continue [7]; on peut aussi l'exprimer sous forme d'une fonction discontinue [10] ou même d'une somme de fonctions $\delta$ du type

$$
n_{\mathrm{ap}}(E)=\sum_{i} \omega_{i} \delta\left(E-E_{i}\right)
$$

où $\omega_{i}$ est le poids du niveau $E_{i}$ dans la densité d'états. Pour des amas d'atomes, dont le nombre de niveaux est petit, c'est évidemment cette dernière expression que l'on emploiera.

Les moments $\mu_{n}$ de la densité d'états exacte $n(E)$ sont définis, rappelons-le, par

$$
\mu_{n}=\int n(E)\left(E-E_{0}\right)^{n} \mathrm{~d} E
$$

où $E_{0}$ est une énergie origine. Si dans la définition (2), on introduit l'expression (1), on obtient des relations entre $\mu_{n}$ et les $\left(\omega_{i}, E_{i}\right)$ de la forme

$$
\mu_{n}=\sum_{i \leqslant P} \omega_{i}\left(E_{i}-E_{0}\right)^{n}
$$

La limite $P$ indique que l'on connaît $2 P$ moments et qu'il y a $2 P$ relations du type (3) qui permettent de déterminer $P$ couples $\left(\omega_{i}, E_{i}\right)$.

Prenons par exemple, le cas d'un agglomérat de $N$ atomes sur lesquels sont centrées $N$ orbitales de type s. Il peut être décrit par un hamiltonien de Hückel de la forme

$$
H=\sum_{i, j} \beta_{i j}|i><j|
$$

où les $\mid i>$ sont les orbitales atomiques $\mathrm{s}$, en prenant l'origine des énergies au niveau atomique s de l'élément ; $\beta_{i j}=\beta$ est l'intégrale de transfert habituelle de la théorie de Hückel, où $i$ et $j$ sont des atomes premiers voisins. Dans la suite des calculs, (4) ne comportera désormais que le seul paramètre $\beta$.

Les $N$ niveaux d'énergie seront intégralement connus si $2 N$ moments $(P=N)$ le sont $\left({ }^{1}\right)$. Si l'on connaît seulement un nombre de moments inférieur $(P<N)$, on pourra toujours utiliser un système d'équations du type (3), mais on n'obtiendra qu'une densité d'états approchée (caractérisée par des poids $\omega_{i}$ non entiers).

La méthode employée pour résoudre le système (3) a été exposée en détail par Gaspard [10] et nous avons à partir de là établi un programme de détermination des $\left(\omega_{i}, E_{i}\right)$. Rappelons pour terminer que dans le cas des agrégats d'atomes, les moments $\mu_{n}$ sont simplement

( ${ }^{1}$ ) Dans le cas où certains niveaux sont dégénérés, ce qui arrive notamment lorsqu'on étudie des agrégats de haute symétrie, un nombre inférieur de moments suffit pour déterminer exactement le spectre de niveaux. Les poids $\omega_{i}$ sont alors des entiers supérieurs à 1 . représentés par la trace de la matrice $H^{n}$, la matrice $H$ étant obtenue par projection de l'opérateur $H$ (formule (4)) sur la base des $\mid i>$, et que l'on peut ainsi en déterminer exactement un grand nombre.

3. Utilisation de la méthode des moments pour la détermination de la géométrie la plus stable d'un amas. - Nous allons examiner ici un problème propre à la Physique des gros amas, à savoir la comparaison entre les stabilités des symétries cubique ou icosaédrique pour des agrégats de 13,19 et 55 atomes dont les formes sont représentées dans la référence [11].

Comme nous l'avons vu plus haut, la méthode des moments permet d'obtenir un spectre de niveaux approché. Nous analyserons 3 paramètres issus de ce spectre (dans le cas d'une fonction d'onde $s$ et d'un électron par atome comme indiqué plus haut) : l'énergie de cohésion $D$, la position de la limite inférieure du spectre de niveaux $E_{1}$ et celle du niveau de Fermi $E_{\mathrm{F}}$ de l'amas, c'est-à-dire du dernier niveau occupé, et nous examinerons principalement avec quelle rapidité ces grandeurs tendent vers leur limite Hückel. On sait que le niveau $E_{1}$ présente un intérêt tout particulier dans le cas d'un amas ferromagnétique à la limite des énergies intra-atomiques de corrélation infinies puisqu'il représente alors l'énergie totale de cet amas [12, 13].

Les résultats sont présentés dans les tableaux I à III que nous allons commenter. Précisons que dans le cas des structures icosaédriques il existe en quelque sorte deux types de premiers voisins : ainsi, pour l'icosaèdre centré de 13 atomes, si on appelle $a$ la distance entre chacun des atomes de surface, c'est-à-dire la longueur de l'arête de l'icosaèdre, la distance entre l'un des atomes de surface et l'atome central est de 0,951 $a$. Nous avons alors défini comme premiers voisins aussi bien les atomes distants de $a$ que de 0,951 $a$.

3.1 ENERGIE DE COHÉSION. - Pour les molécules de 13 et 19 atomes, on voit qu'il faut pousser les calculs jusqu'à un ordre en moments assez élevé pour obtenir le bon classement des stabilités. En effet, 10 moments sont nécessaires dans le premier cas et 18 dans le deuxième. Par contre, pour les molécules de 55 atomes, on voit que dès l'ordre 8 , on obtient le classement correct. Signalons cependant que le cas des amas de 55 atomes se montre ici plus favorable comme nous l'avons dit plus haut à cause de la haute symétrie des formes choisies, donc des nombreuses dégénérescences (il y a seulement 17 racines distinctes pour l'icosaèdre et 18 pour le cuboctaèdre) qui diminuent d'autant le nombre total de moments à connaître pour obtenir exactement les niveaux d'énergie Hückel.

On se trouve donc ici dans un cas où la méthode des moments permet d'économiser du temps de calcul à la machine. En effet, le calcul des moments et la recherche des $\left(\omega_{i}, E_{i}\right)$ jusqu'à l'ordre $12\left(^{2}\right)$ pour 55 atomes

$\left(^{2}\right)$ On doit en effet s'assurer qu'on a bien atteint le classement limite des stabilités en prenant comme critère que le même classement apparaît par exemple à 3 ordres d'approximation successifs. On arrive ainsi à 12 moments pour les amas de 55 atomes. 
TABLEAU I

Comparaison de l'énergie électronique obtenue avec $2 P$ moments $(2 P=4$ à 10 et 12$)$ au résultat Hückel (unités $\beta, \beta<0$ ) pour les molécules de 13 atomes (formes de la référence [11]).

Les signes + et - de la colonne de droite indiquent à partir de quelle valeur de $2 P$ on retrouve le classement des énergies des 3 amas donné par le calcul Hückel.

$E_{1}$ : limite inférieure de la bande de niveaux d'énergie (unités $\beta$ ); $E_{\mathrm{F}}$ : dernier niveau occupé (unités $\beta$ ).

Par suite des dégénérescences dues aux symétries (voir texte), on trouve l'énergie Hückel exacte avec respectivement 8 moments pour le cuboctaèdre et 10 pour l'icosaèdre.

\begin{tabular}{|c|c|c|c|c|c|}
\hline$N=13$ & $2 P$ & Icosaèdre centré & Cuboctaèdre & Hcp unit cell & $\begin{array}{l}\text { Ordre } \\
\text { Hückel }\end{array}$ \\
\hline - & - & - & - & - & - \\
\hline \multirow{4}{*}{$D$} & 4 & 17,170155 & 17,829779 & 17,829779 & - \\
\hline & 6 & 23,022483 & 24,651158 & 25,129856 & - \\
\hline & 8 & 21,808941 & $24,000 \quad$ (exacte) & 21,441048 & - \\
\hline & 10 & 21,960412 (exacte) & 24,000 & 22,493394 & + \\
\hline \multicolumn{2}{|c|}{$\begin{array}{l}\text { Valeur } \\
\text { Hückel }\end{array}$} & $\begin{array}{l}21,960412 \\
\text { (5 racines distinctes) }\end{array}$ & $\begin{array}{l}24,000 \\
(4 \text { racines distinctes })\end{array}$ & $\begin{array}{c}22,628541 \\
\text { (8 racines distinctes) }\end{array}$ & + \\
\hline \multirow{4}{*}{$E_{1}$} & 4 & 4,892210 & 4,038188 & 4,038188 & \\
\hline & 6 & 6,607018 & 5,856031 & 5,750772 & \\
\hline & 8 & 6,771850 & 6,000 & 5,996369 & \\
\hline & 10 & 6,772002 & 6,000 & 5,999939 & \\
\hline \multicolumn{2}{|c|}{ Valeur Hückel } & 6,772002 & 6,000 & 6,000 & \\
\hline & 4 & $-1,32078$ & $-1,37152$ & $-1,37152$ & \\
\hline & 6 & $-1,77096$ & $-1,89624$ & $+1,03131$ & \\
\hline & 8 & $-1,03636$ & 0 & $-0,73348$ & \\
\hline & 10 & $-1,000 \quad$ (exacte) & 0 & $-0,38375$ & \\
\hline & 12 & $-1,000$ & 0 & $-0,73562$ & \\
\hline Valeur I & kel & $-1,000$ & 0 & $-0,57199$ & \\
\hline
\end{tabular}

demande $6,03 \mathrm{~s}$ (icosaèdre) et $6,23 \mathrm{~s}$ (cuboctaèdre) au lieu de 9,88 et 9,02 s respectivement pour la diagonalisation de la matrice de Hückel.

Remarquons au passage que dans le cas présent où l'on n'a pas cherché à minimiser $D$ en faisant varier les distances entre atomes (pas de relaxation des amas), la structure icosaédrique, la moins stable pour 13 atomes, correspond au contraire à une plus forte cohésion dès que $N$ augmente (pour $N=19$ et 55 atomes).

3.2 Précision sur Les Niveaux. - Pour tous les types de molécules étudiés, la rapidité de la convergence vers la limite Hückel exacte est assez grande pour $E_{1}$ (tableaux I à III). La valeur du niveau $E_{1}$ est atteinte avec une précision $\delta_{\mathrm{r}}$ meilleure que $10^{-3}$ dès l'ordre 8 pour les 3 molécules de 13 atomes, l'ordre 10 pour les 2 molécules de 19 atomes et l'ordre 12 pour les 2 molécules de 55 atomes [5].

Par contre, on peut observer qu'il est loin d'en être de même pour le dernier niveau occupé $E_{\mathrm{F}}$ qui se situe en milieu de bande. En effet, la variation de la position de $E_{\mathrm{F}}$ quand le nombre $2 P$ de moments connus augmente est caractérisée par de fortes oscillations qui s'amortissent relativement lentement et la limite Hückel paraît difficile à atteindre avec une précision correcte.

4. Utilisation de la méthode des moments pour la détermination de l'évolution avec le nombre d'atomes de l'énergie de cohésion. - Nous nous intéressons ici au cas particulier de molécules linéaires de $N=10$ atomes ou moins. 


\section{TABLEAU II}

Même calcul pour 19 atomes qu'au tableau I. Pour $E_{1}$ et $E_{\mathrm{F}}$, on donne l'erreur $\delta_{\mathrm{r}}$ pour diverses valeurs de $2 P$.

\begin{tabular}{|c|c|c|c|c|c|c|}
\hline$N=19$ & $2 P$ & \multicolumn{2}{|c|}{ Double icosaèdre } & \multicolumn{2}{|c|}{ Octaèdre c.f.c. } & $\begin{array}{c}\text { Ordre } \\
\text { Hückel }\end{array}$ \\
\hline \multirow{6}{*}{$D$} & 8 & \multicolumn{2}{|c|}{35,582307} & \multicolumn{2}{|c|}{32,423859} & + \\
\hline & 10 & \multicolumn{2}{|c|}{34,215625} & \multicolumn{2}{|c|}{36,550724} & - \\
\hline & 12 & \multicolumn{2}{|c|}{35,256696} & \multicolumn{2}{|c|}{35,510750} & - \\
\hline & 14 & \multicolumn{2}{|c|}{36,188271} & \multicolumn{2}{|c|}{35,996142} & + \\
\hline & 16 & \multicolumn{2}{|c|}{35,651470} & \multicolumn{2}{|c|}{36,142570 (exacte) } & - \\
\hline & 18 & \multicolumn{2}{|c|}{36,436317} & \multicolumn{2}{|c|}{36,142570} & + \\
\hline \multicolumn{2}{|c|}{$\begin{array}{l}\text { Valeur } \\
\text { Hückel }\end{array}$} & \multicolumn{2}{|c|}{$\begin{array}{c}36,281347 \\
\text { (13 racines distinctes) }\end{array}$} & \multicolumn{2}{|c|}{$\begin{array}{l}36,142570 \\
\text { (8 racines distinctes) }\end{array}$} & + \\
\hline \multirow{6}{*}{$E_{1}$} & 8 & 7,576314 & $\delta_{\mathrm{r}}: 9,5 \times 10^{-3}$ & 6,860483 & $\delta_{\mathrm{r}}: 6,4>$ & \\
\hline & 10 & 7,635293 & $\delta_{\mathrm{r}}: 1,05 \times 10^{-3}$ & 6,898411 & $\delta_{\mathrm{r}}: 9,5>$ & \\
\hline & 12 & 7,642361 & $\delta_{\mathrm{r}}: 4,1 \times 10^{-5}$ & 6,898969 & & \\
\hline & 14 & 7,642621 & $\delta_{\mathrm{r}}: 3 \times 10^{-7}$ & 6,898979 & & \\
\hline & 16 & 7,642623 & & 6,898980 & & \\
\hline & 18 & 7,642623 & & 6,898980 & & \\
\hline
\end{tabular}

\begin{tabular}{|c|c|c|c|c|}
\hline \multicolumn{2}{|c|}{ Valeur Hückel } & \multicolumn{2}{|c|}{7,642623} & \multirow{2}{*}{$\begin{array}{r}6,898980 \\
-4,017891\end{array}$} \\
\hline \multirow{7}{*}{$E_{\mathrm{F}}$} & 8 & $-0,206929$ & & \\
\hline & 10 & $-1,058781$ & & $+0,279204$ \\
\hline & 12 & $-1,409940$ & & $-0,549682$ \\
\hline & 14 & $-0,847270$ & & $-0,046844$ \\
\hline & 16 & $-1,171656$ & & 0 \\
\hline & 18 & $-1,551371$ & $\delta_{\mathrm{r}}: 25,6 \times 10^{-2}$ & 0 \\
\hline & 20 & $-1,295765$ & $\delta_{\mathrm{r}}: 4,9 \times 10^{-2}$ & 0 \\
\hline \multicolumn{2}{|c|}{ Valeur Hückel } & $-1,235403$ & & 0 \\
\hline
\end{tabular}

Partant du même principe qu'au paragraphe précédent, nous avons cherché jusqu'à quel ordre en moments il convenait de poursuivre les calculs pour voir apparaître un phénomène intéressant manifesté par ces molécules, c'est-à-dire la présence d'alternances de l'énergie de cohésion avec la parité de $N$ (énergie de liaison par atome plus élevée pour les molécules neutres de $N$ pair que pour celles de $N$ impair [5, 14]).

Les résultats sont récapitulés dans le tableau IV dans lequel nous donnons les quantités

$$
\Delta_{N}=D_{\mathrm{e}}(N)-D_{\mathrm{e}}(N-1),
$$

différences entre les énergies Hückel des chaînes de $N$ et $N-1$ qui mettent mieux en évidence les alternances que les énergies de liaison par atome ellesmêmes. On sait que les alternances observées lorsque la parité de $N$ varie se traduisent par $\Delta_{N}>\Delta_{N-1}$ si $N$ est pair [5] (grandeurs soulignées tableau IV). On voit qu'avec la méthode des moments, il faut pratiquement aller jusqu'à la connaissance complète du spectre $(P=N)$ pour retrouver l'effet d'alternance dans le bon sens.

On pouvait légitimement s'attendre à ces difficultés d'après les remarques du paragraphe précédent. Il s'agit en effet de molécules linéaires dont le spectre de niveaux ne présente aucune dégénérescence (pas de haute symétrie). Donc le niveau $E_{\mathrm{F}}$ ne peut être que très mal connu sauf si $P=N$, et comme il a été montré dans d'autres articles [15] que, dans l'approximation de Hückel, c'est précisément la position du niveau $E_{\mathrm{F}}$ qui joue un rôle déterminant dans l'interprétation des alternances, on est ici en présence d'une propriété où la méthode des moments est visiblement mal adaptée. 


\section{TABLEAU III}

Même calcul pour 55 atomes qu'au tableau $\mathrm{I}$. On donne l'erreur relative $\delta_{\mathrm{r}}$ pour l'énergie électronique totale $D, E_{1}$ et $E_{\mathrm{F}}$. On indique également le temps en secondes mis par les deux méthodes, Hückel et moments.

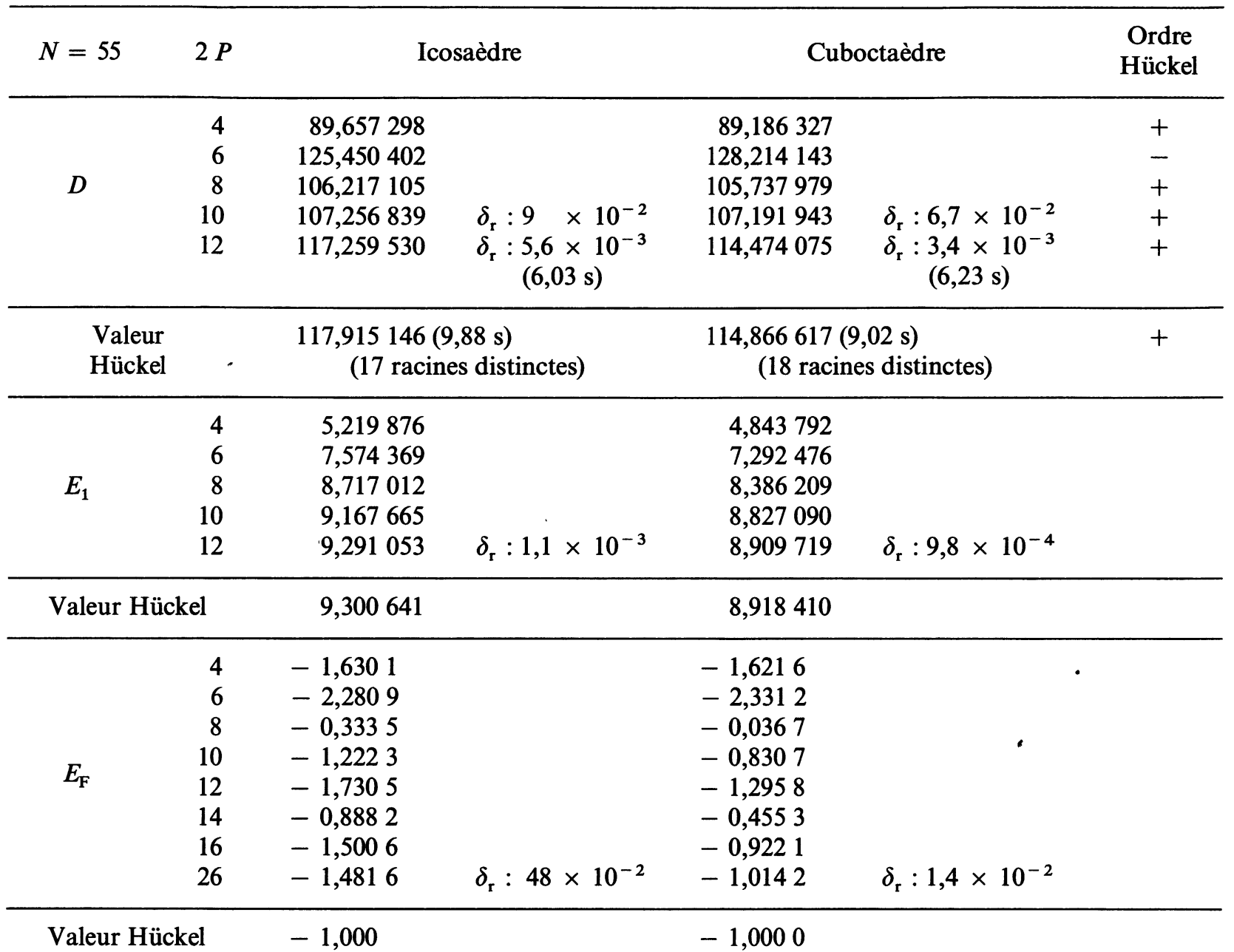

TABLEAU IV

Evolution des $\Delta_{N}$ (en unités $\beta, \beta<0$ ) pour les molécules linéaires de $N$ atomes en fonction du nombre $2 P$ de moments utilisé.

Comparaison aux alternances trouvées par la méthode de Hückel $(2 P=2 N)$ (voir texte). Le signe - de la dernière ligne indique qu'on ne trouve pas les bonnes alternances avec la valeur de $2 P$ placée en tête de la colonne correspondante.

\begin{tabular}{|c|c|c|c|c|c|}
\hline \multirow[b]{2}{*}{$2 P$} & \multicolumn{5}{|c|}{$\Delta_{N}$} \\
\hline & $\begin{array}{c}2 N \\
\text { (Hückel) }\end{array}$ & $2 N-2$ & $2 N-4$ & $2 N-6$ & $2 N-8$ \\
\hline - & - & - & - & - & - \\
\hline 4 & 1,644 & 0,464 & - & - & - \\
\hline 5 & $\overline{0,992}$ & 1,870 & 0,161 & - & - \\
\hline 6 & 1,524 & 0,870 & 2,063 & $-0,123$ & - \\
\hline 7 & 1,067 & 1,618 & 0,776 & 2,221 & $-0,398$ \\
\hline 8 & 1,463 & 1,005 & 1,689 & 0,691 & 2,384 \\
\hline 9 & $\overline{1,110}$ & 1,514 & 0,960 & 1,753 & 0,610 \\
\hline 10 & 1,425 & - & 1,552 & 0,918 & 1,815 \\
\hline
\end{tabular}

Ordre par rapport à Hückel 
5. Conclusion. - Nous avons transposé la méthode des moments au cas d'amas finis d'atomes. Nous concluons de cette étude que les performances de cette méthode sont notables (et pourraient sans doute être encore améliorées si le programme de calcul était optimisé) tant pour l'examen des stabilités relatives de gros amas ( $\sim 55$ atomes) que pour la détermination $\mathrm{du}$ niveau de bas de bande.

On a vu que le gain de temps de calcul par cette méthode était important surtout lorsqu'il y avait des dégénérescences de niveaux d'énergie, dégénérescences dues à la haute symétrie des molécules étudiées. Il faudra donc, dans les améliorations projetées, conserver cette caractéristique des amas. Ceci nous conduit à envisager deux types d'études.

D'une part, dans les structures icosaédriques, on peut introduire plusieurs paramètres $\beta$ selon que les atomes premiers voisins sont à la distance $a$ ou $0,951 a$ (on pourrait même introduire d'autres paramètres $\beta$ pour les deuxièmes, troisièmes, etc..., voisins).

On peut d'autre part envisager une étude de type self-consistent. Rappelons que dans ces méthodes (par exemple la méthode CNDO [16] ou celle plus approchée exposée par Lasarov et al. [2]), la prise en compte du terme de répulsion coulombienne revient à introduire une dépendance des $\beta_{i j}$ de l'hamiltonien avec la charge localisée sur les atomes $i, j$ et leurs voisins. Les charges localisées n'étant connues qu'après résolution de l'hamiltonien, on voit que l'on est conduit à un traitement itératif : on fixe $a$ priori une première répartition de charge, on calcule les $\beta$ par les formules appropriées (voir [16, 2]); on en déduit une nouvelle répartition et on réitère le processus jusqu'à la convergence.

Nous ne nous sommes intéressés dans cet article qu'à la densité d'états totale; cependant la méthode des moments permet aussi d'atteindre aisément la densité d'états localisée [17]; il suffit pour cela d'introduire dans le système (3), non pas les traces des matrices $H^{n}$, mais pour un atome $i$ donné, les éléments de matrice $\left(H^{n}\right)_{i i}$. On comprend que, comme pour les calculs classiques self-consistents décrits plus haut, on puisse procéder avec la méthode des moments par itérations successives en déduisant à chaque fois des densités d'états locales les nouveaux paramètres de l'hamiltonien. Dans ce type de calcul, la symétrie n'est évidemment pas détruite et on peut prévoir que les gains de temps de calcul devraient s'ajouter à chaque itération.

Ces deux applications projetées de la méthode des moments tendent donc vers une description plus proche de la réalité que le cas traité ici.

Un autre objectif est aussi d'étendre les calculs à des amas de quelques centaines d'atomes où il existe des résultats expérimentaux $[18,19]$ et où la recherche des géométries les plus stables a déjà été faite dans l'hypothèse où les atomes interagissent par des potentiels du type Van der Waals [11, 20].

\section{Bibliographie}

[1] IтоH, H., J. Phys. F 4 (1974) 1930.

[2] Lasarov, D., Markov, P., Surf. Sci. 14 (1969) 320.

[3] KuBo, R., J. Phys. Soc. Japan 17 (1962) 975.

[4] Denton, R., Muhlschlegel, B., Scalapino, D. J., Phys. Rev. B 7 (1973) 3589.

[5] Leleyter, M., Thèse d'Etat (1975) Orsay.

[6] Voir par exemple Salem, L., The Molecular Orbital Theory of Conjugated Systems (Benjamin, New York) 1966.

[7] Cyrot-Lackmann, F., Thèse d'Etat (1968) Orsay.

[8] Friedel, J., J. Less Comm. Metals 28 (1972) 241.

[9] Ducastelle, F., J. Physique Colloq. 35 (1974) C7-79.

[10] Gaspard, J.-P., Thèse 3e Cycle (1972) Orsay.

[11] Hoare, M. R., Pal, P., Adv. Phys. 20 (1971) 161 ; Nature, Phys. Sci. 236 (1972) 35.
[12] Joyes, P., Leleyter, M., Lederer, P., Heritier, M., J. Physique 36 (1975) 411.

[13] Nagaoka, Y., Phys. Rev. 147 (1966) 392.

[14] Baetzold, R. C., J. Chem. Phys. 55 (1971) 4363.

[15] Joyes, P., J. Phys. Chem. Sol. 32 (1971) 1269.

[16] Pople, J. A., Beveridge, D. L., Approximate Molecular Orbital Theory (McGraw Hill, New York) 1970.

[17] Cyrot-Lackmann, F., Desjonqueres, M. C., Gaspard, J. P., J. Phys. C 7 (1974) 925.

[18] Dixmier, J., J. Physique Colloq. 36 (1975) C2-65.

[19] Taupin, Ch., J. Phys. \& Chem. Sol. 28 (1967) 41.

[20] Burton, J. J., J. Chem. Phys. 52 (1970) 345; Nature 229 (1971) 335 . 